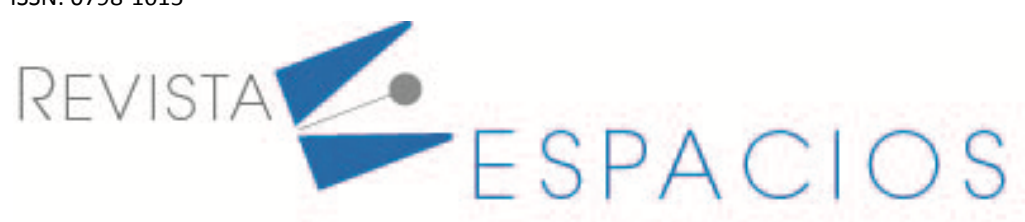

\title{
Nivel de estrés y rendimiento académico en estudiantes universitarios que trabajan y los que no
}

\section{Level of stress and academic performance in college students who work and who don't}

LICHT-ARDILA, Valentina ${ }^{1}$

SOTO-GUALDRÓN, Silvia N. ${ }^{2}$

ÁNGULO-RINCÓN, Rosalba ${ }^{3}$

\begin{abstract}
Resumen
Algunos estudiantes universitarios tienen la necesidad de asumir un trabajo para tener una mejor calidad de vida, por lo que se ven expuestos a largas jornadas tanto laborales como académicas, incrementando así los niveles de estrés. Por esto, el presente estudio correlacional, identificó las diferencias que existen en el estrés y el rendimiento académico entre estudiantes que trabajan y los que no; encontrando un nivel moderado de estrés y promedios académicos similares en ambos grupos.

Palabras clave: estrés académico, rendimiento, trabajo y no trabajo.
\end{abstract}

\begin{abstract}
Some university students have the need to assume a job to have a better quality of life, so they are exposed to long working and academic hours, increasing their stress levels. For this reason, this correlation research, identifies the differences between stress and academic performance from a working students group and non-working students group, finding a moderate level of stress and similar academic averages in both groups.

Key words: academic stress, performance, work and no work.
\end{abstract}

\section{Introducción}

\subsection{Estrés}

A través de los años, la importancia del estrés en la sociedad ha ido aumentando, al igual que las investigaciones acerca de este fenómeno, el cual se define como la respuesta inespecífica del organismo a toda exigencia hecha sobre él (Selye, 1956). Esto se debe a que en el mundo "uno de cada cuatro individuos sufre de algún problema grave de estrés y en las ciudades, se estima que el 50 por ciento de las personas tienen algún problema de salud mental de este tipo" (Caldera, Pulido y Martínez, 2007, p. 78). Por lo que se considera un aspecto de gravedad, puesto que, el estrés es un importante generador de diversas patologías (Berrío y Mazo, 2011).

Además, el estrés se presenta en diversos ámbitos, tales como el académico y laboral. Entendiendo el primero como aquél que se genera debido a las demandas que se imponen en el área educativa (Caldera et al., 2007), de

\footnotetext{
${ }^{1}$ Estudiante de pregrado. Psicología. Universidad Pontifica Bolivariana. valentina.licht.2017@upb.edu.co

2 Estudiante de pregrado. Psicología. Universidad Pontifica Bolivariana. silvia.soto.2017@upb.edu.co

${ }^{3}$ Docente adjunto. Facultad de Psicología. Universidad Pontificia Bolivariana. rosalba.angulo@upb.edu.co
} 
forma individual y grupal, tales como: Trabajos, lecturas, talleres, exámenes; y el segundo, como un estrés dependiente de las demandas del trabajo y de los factores moderadores de las mismas, que incluye la idea en el empleado de no estar a la altura de las actividades cotidianas propias de su puesto, provocando así, sensaciones de ansiedad, temor, frustración y enojo (Ángeles y Gutiérrez, 2012).

\subsection{Rendimiento académico}

El rendimiento académico ha sido definido de diferentes formas. Jiménez (2000), citado por Edel, (2003, pp. 2), postula que este es un nivel de conocimientos demostrado en un área o materia, comparado con la norma de edad y nivel académico general. Sin embargo, las expectativas de docentes, familia y los pares también se definen como variables para medir el rendimiento de un escolar (Edel, 2003).

Se caracteriza por ser el resultado de las calificaciones del estudiante, como respuesta a los estímulos que recibe del contexto en el que se desarrolla, tanto educativo como social. Asimismo es una evaluación de los logros de aprendizaje del estudiante y la manera como lo asimila, se deben tener en cuenta conocimientos, habilidades y actitudes para comprobar lo aprendido (Sotelo y Gutiérrez, 2020).

\subsection{Estrés y rendimiento académico}

En la actualidad, es común encontrar jóvenes universitarios que están expuestos tanto a estrés académico como estrés laboral (Francisco-Méndez, 2015), puesto que adquirir ambas responsabilidades, trabajar y estudiar, en la mayoría de los casos, se convierte en una necesidad y una oportunidad para mejorar sus condiciones de vida.

De ahí la importancia de identificar la relación entre el rendimiento académico y el estrés laboral de estudiantes universitarios que trabajan. La revisión de la literatura expone pocos estudios que abordan la relación entre estas dos variables, son incluso inexistentes. Con respecto a esto, Carrillo y Ríos (2013) encontraron que las horas de trabajo reducen el tiempo con el que cuentan los estudiantes para cumplir con sus responsabilidades académicas y, por ende, incide de manera negativa en su rendimiento académico. Así también, DeSimone (2008) evidenció que el desempeño de los estudiantes se ve afectado por el número de horas que estos deban trabajar.

Por otra parte, Caballero (2006) reporta un estudio comparativo entre aquellos que trabajan y los que no trabajan, analizando el burnout ${ }^{4}$, el engagement ${ }^{5}$ y el rendimiento académico, dirigido a 202 jóvenes estudiantes de segundo a décimo semestre de la jornada nocturna del programa de Psicología de una universidad privada de la ciudad de Barranquilla. En la investigación se encontró que los estudiantes que trabajan se perciben más autoeficaces y dedicados que quienes no lo hacen. Asimismo, no se presentaron diferencias en el promedio académico ni en el número de semestres perdidos entre los dos grupos, sin embargo, este estudio mostró que los estudiantes que no trabajan son quienes pierden un mayor número de exámenes.

En otra investigación, realizada en la Universidad Autónoma de Yucatán, Domínguez, Guerrero y Domínguez (2015), plantearon como objetivo conocer aquellos factores que pueden generar estrés en el ámbito escolar y cómo este se relaciona con el rendimiento académico, para lo cual, se administró el inventario SISCO del Estrés Académico a estudiantes de sexto semestre de la Licenciatura en Educación. Con respecto a lo anterior, se encontró que el estrés no afecta de manera significativa el rendimiento de los estudiantes, sin embargo, éste

\footnotetext{
${ }^{4}$ Burnout o síndrome del quemado es definido como la inadecuada forma de afrontar el estrés crónico, cuyos síntomas principales son la despersonalización, el agotamiento emocional y la disminución del desempeño personal. (Saborío e Hidalgo, 2015)

${ }^{5}$ Engagement se define como un estado psicológico positivo, persistente, motivacional y afectivo, de realización en los empleados. (Maslach, Schaufeli y Leiter, 2001).
} 
interfiere en la realización de algunas de sus actividades académicas, tales como realizar trabajos, exámenes, exposiciones, entre otros.

Estudios recientes, como el de Almeida y Lasluisa (2020), buscaba determinar la relación existente entre el estrés y el rendimiento académico en estudiantes, por lo que se llevó a cabo un estudio descriptivo correlacional y de corte transversal, en el que se aplicó el Inventario SISCO de Estrés Académico y la Escala de Autoestima en adolescentes. Con respecto al estrés académico, se evidenció que se presenta en niveles moderados; y en cuanto a la relación de este con el rendimiento académico, se encontró que es nula, es decir, el estrés académico no es un predictor del rendimiento académico.

Teniendo en cuenta lo anterior, se propone comparar los niveles de estrés académico y analizar la relación entre el nivel de estrés y desempeño académico entre un grupo de estudiantes que trabajan y no trabajan de la Universidad Industrial de Santander y la Universidad Pontificia Bolivariana. Para ello se determinan los niveles de estrés presentes en los participantes y se hace un reconocimiento para comprobar si existe o no relación entre el estrés y el rendimiento académico.

\section{Metodología}

Según el nivel de medición y el análisis de la información, la investigación es de enfoque cuantitativo con diseño no experimental de tipo comparativo y correlacional donde se pretende identificar las diferencias entre dos variables, estrés y rendimiento académico, en estudiantes que trabajan y no trabajan de la Universidad Industrial de Santander y la Universidad Pontificia Bolivariana de la ciudad de Bucaramanga (Colombia).

Se realizó un muestreo no probabilístico intencional o por conveniencia de los estudiantes que trabajan y no trabajan de la Universidad Pontificia Bolivariana, seccional Bucaramanga y la Universidad Industrial de Santander, obteniendo un total de 164 estudiantes voluntarios para la aplicación del Inventario SISCO y de un cuestionario de datos sociodemográficos. Dicha participación se realizó de manera presencial a 41 estudiantes, mientras que otros 123 estudiantes, en el marco de la pandemia del COVID-19, participaron de manera virtual; teniendo en cuenta como criterio de inclusión: estudiantes que fueran mayores de edad y que estuvieran activos durante el primer semestre del 2020, cursando quinto semestre o superior de algún programa ofrecido por ambas universidades.

En cuanto a la aplicación del cuestionario de datos sociodemográficos, se incluyeron datos de los estudiantes, tales como: Universidad, edad, género, estado civil, tipo de vivienda y semestre; incluyendo un ítem donde podían referir si trabajan o no, así como el tiempo que llevaban trabajando y el tipo de trabajo. Así también, se implementó el Inventario SISCO de estrés académico que tiene como principal objetivo reconocer las características del estrés que suele acompañar a los estudiantes de educación media superior, superior y posgrado; a partir del abordaje de tres dimensiones: Estresores, síntomas y estrategias de afrontamiento, de siete ítems cada una, para un total de veintiún ítems. El inventario en su totalidad presenta una confiabilidad en alfa de Cronbach de .85. La dimensión de estresores presenta una confiablidad de .83; la de síntomas presenta una confiablidad de .87; y la de estrategias de afrontamiento una de .85. Todas ellas también en alfa de Cronbach. Con respecto a la consistencia interna, los resultados muestran que todos los ítems correlacionaron positivamente con el puntaje global obtenido por cada encuestado (Barraza, 2018). La validación en Colombia se llevó a cabo con estudiantes de la Universidad Pontificia Bolivariana, seccional Bucaramanga y de la Universidad Industrial de Santander UIS a partir del programa SPSS 10 y se obtuvo un nivel de fiabilidad de 0.8 (Cáceres \& Peña, 2008). 


\section{Resultados}

En la Tabla 1, se muestran los resultados relacionados con la aplicación del cuestionario sociodemográfico, tanto de estudiantes que trabajan como de estudiantes que no.

Tabla 1

Información sociodemográfica de la muestra

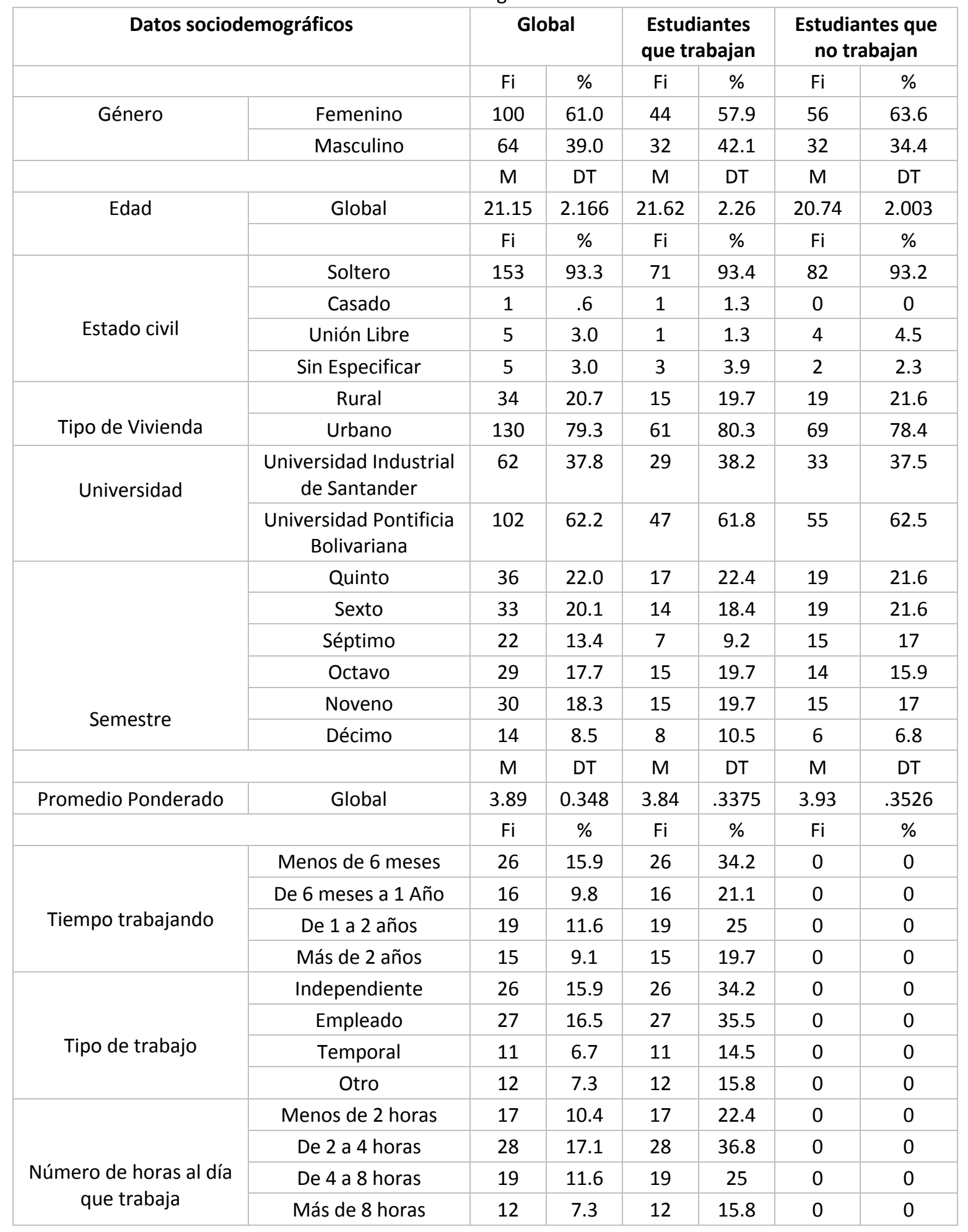


En cuanto a los datos sociodemográficos, se puede observar que el $61 \%$ fueron mujeres y el $39 \%$ hombres, con una edad media global de 21.15 años. Asimismo, el 57.9\% de los estudiantes que trabajan fueron del género femenino y el $42.1 \%$ del género masculino, con promedio de edad de 21.62 años. Respecto a los estudiantes que no trabajan, el $63.6 \%$ correspondieron a mujeres, y el $34.4 \%$ fueron hombres, con una edad media de 20.74 años.

Con relación al estado civil, se evidencia que la mayoría de los participantes son solteros (93.3\%). Además, respecto al tipo de vivienda, prevalece el urbano con un $79.3 \%$ frente al rural con un $20.7 \%$.

Por otro lado, el $37.8 \%$ de los estudiantes pertenecen a la Universidad Industrial de Santander, de los cuales el $46.77 \%$ trabajan y el $53.23 \%$ no trabajan; así también, el $62.2 \%$ son estudiantes de la Universidad Pontifica Bolivariana, donde el $46 \%$ trabajan y el $53.92 \%$ no trabajan. En cuanto al rendimiento académico de los mismos, se encontró que la media global del promedio ponderado es de 3.89; el de los estudiantes que trabajan es de 3.84 y el de los que no trabajan es de 3.93. Teniendo en cuenta que la escala de valoración académica implementada en dichas instituciones es de 0.0 a 5.0.

\subsection{Niveles de estrés}

Teniendo en cuenta los baremos del inventario SISCO (Ver tabla 2), se puede constatar que tanto los estudiantes que trabajan como los que no trabajan presentan un nivel de estrés moderado, evidenciando en un $62.1 \%$ en el primer grupo y $60.5 \%$ en el segundo grupo, respectivamente (Ver tabla 3 ).

Tabla 2

Baremo indicativo centrado en el valor teórico de la variable

\begin{tabular}{|c|c|}
\hline Valor & Nivel de estrés \\
\hline De 0 a $33 \%$ & Leve \\
\hline De $34 \%$ a $66 \%$ & Moderado \\
\hline De $67 \%$ al $100 \%$ & Severo \\
\hline
\end{tabular}

Tabla 3

Media de estrés académico presentado por los estudiantes

\begin{tabular}{|c|c|c|c|c|}
\hline & Estudiantes que no trabajan & $\%$ & Estudiantes que trabajan & $\%$ \\
\hline Puntuación Global & 3.025 & 60.5 & 3.105 & 62.1 \\
\hline Estresores & 3.151 & 63 & 3.180 & 63.6 \\
\hline Síntomas & 2.866 & 57.32 & 2.981 & 59.62 \\
\hline Estrategias & 3.058 & 61.16 & 3.156 & 63.12 \\
\hline
\end{tabular}

La distribución de los datos del total de estrés académico es normal, puesto que el valor de significancia es mayor a .05 (.200).

\subsection{Estrés y rendimiento académicos}

En la tabla 4 se presenta la correlación de Spearman entre estrés y rendimiento académico. El valor del coeficiente de correlación (-.006) indica que la relación entre el estrés y el rendimiento académicos es casi nula, sin embargo, al ser un valor negativo, permite afirmar que, a mayor estrés, menor es el rendimiento académico presentado por los estudiantes. 
Tabla 4

Correlación (Spearman) entre estrés

y rendimiento académico

\begin{tabular}{|c|c|c|}
\hline & SISCO & \\
\hline Promedio ponderado & Coeficiente de correlación & -.006 \\
\hline & Sig. (Bilateral) & .939 \\
\hline
\end{tabular}

Con respecto a la tabla 5, el valor de la significancia es mayor a 0.05 , entonces se rechaza la hipótesis alterna, quedando así la hipótesis nula que indica que no existe diferencias en el estrés académico entre estudiantes que trabajan y que no trabajan.

Tabla 5

Prueba de

muestras independientes

\begin{tabular}{|c|c|c|c|c|c|c|}
\hline & $\begin{array}{c}\text { Prueba de Levene } \\
\text { de igualdad de } \\
\text { varianzas }\end{array}$ & $\begin{array}{c}\text { Prueba t para la } \\
\text { igualdad de medias }\end{array}$ & gl & $\begin{array}{c}\text { Sig. } \\
\text { (bilateral) }\end{array}$ \\
\hline & SISCO & $\mathbf{F}$ & Sig. & .211 & .831 & 162 \\
\hline & $\begin{array}{c}\text { Se asumen } \\
\text { varianzas iguales }\end{array}$ & 1.57 & & .818 & 143.766 & .415 \\
\hline & $\begin{array}{c}\text { No se asumen } \\
\text { varianzas iguales }\end{array}$ & & & & \\
\hline
\end{tabular}

\section{Discusión}

Se encontró que, en cuestión de participantes, las mujeres fueron mayoritarias. Asimismo, la media de estrés académico se sitúa en -moderado- en los dos grupos de estudiantes encuestados. La comparación entre los estudiantes que trabajan y los que no trabajan demuestra que no existen diferencias significativas, tanto en el estrés académico como en el rendimiento de estos, sin embargo, la relación entre el estrés y el rendimiento académico es inversa, siendo el estrés un factor predictor de un bajo rendimiento para los estudiantes.

El estrés académico se puede definir como una reacción de activación fisiológica, emocional, cognitiva y conductual ante estímulos y eventos académicos; y cuando se presenta más allá de los niveles óptimos puede agotar las energías del estudiante, deteriorar su desempeño e incluso dañar su salud mental y física (Rivas et al., 2014). En cuanto a los niveles de estrés académico en este estudio, se encontró que tanto los estudiantes que trabajan como los que no trabajan experimentan un nivel moderado de estrés según el baremo indicativo centrado en el valor teórico de la variable, lo cual se relaciona con los resultados obtenidos por Kloster y Perrotta (2019) en su estudio, donde la mayoría de los estudiantes presentan un nivel moderado de estrés.

Por su parte, el rendimiento académico se refiere al producto de la asimilación del contenido de los programas de estudio, que se expresa en calificaciones dentro de una escala convencional (Figueroa, 2004, citado en Salinas y González, 2019). En esta investigación, los estudiantes que no trabajan muestran un promedio más alto en comparación con los estudiantes que trabajan. Esto se relaciona con lo encontrado por Barreto, Celis y Pinzón (2019) donde los estudiantes que estudian y trabajan afirman que los resultados académicos se ven afectados por la falta de tiempo para responder de manera simultánea a las metas laborales y académicas. No obstante, en un estudio realizado por Salinas y González (2019), el promedio académico de los estudiantes que trabajan es 
mayor que el promedio de los estudiantes que no trabajan, empero la diferencia entre dichos promedios no es significativa.

Para los estudiantes universitarios conseguir un trabajo que tenga muy poca relación con su campo académico se ha convertido en una acción común y en una decisión que la mayoría de estos debe tomar, lo cual representa un aumento en la adquisición de responsabilidades que pueden repercutir en su rendimiento, tanto laboral como académico (Neyt, Omey, Verhaest y Baert, 2019).

Con relación a los resultados presentados, se evidencia que coinciden con lo encontrado por Darolia (2014) en una investigación realizada a estudiantes universitarios, en la cual no se encontró evidencia de que las calificaciones se vean afectadas por el trabajo que estos desempeñan fuera de la academia. Asimismo, en el estudio de Nonis y Hudson (2006), los hallazgos demuestran que la cantidad de tiempo dedicado a la actividad laboral por parte de los estudiantes no influye en su rendimiento académico. Por otra parte, se hallaron estudios que difieren de lo encontrado en la presente investigación, como el de Francisco-Mendez (2015) en el cual se determinó que el rendimiento académico de los estudiantes que laboran se ve perjudicado por manejar niveles altos de estrés.

Los resultados del presente estudio se podrían explicar en torno a la pandemia actual y los procesos de adaptación de las personas a los recursos virtuales, así como los cambios en su rutina diaria, tales como trasladar el trabajo y el estudio al hogar, a través de las plataformas virtuales, lo cual implica reajustar el tiempo que se invertía en el transporte de un lugar a otro para la realización de actividades domésticas, así como suprimir los encuentros sociales y la visita a lugares de alta afluencia de público, que permite, posiblemente, disponer de mayor tiempo para las actividades académicas propias de su formación.

\section{Conclusiones}

En general, cuando se comparan los promedios académicos entre los dos grupos de estudiantes universitarios que trabajan y no trabajan de la Universidad Industrial de Santander y la Universidad Pontificia Bolivariana, la diferencia encontrada no es estadísticamente significativa, similitud que puede ser explicada porque, tanto el nivel de exigencia, como los criterios de evaluación de ambas instituciones para algunos de los programas ofertados en estas son similares. Por otro lado, con respecto al estrés académico percibido, se determinó que éste se presenta en un nivel "moderado" en ambos grupos, lo cual se puede explicar porque ambas universidades, a pesar de ser una privada y la otra pública, comparten dinámicas flexibles relacionadas con los horarios, créditos y cursos académicos, lo cual favorece la adquisición de una responsabilidad diferente al estudio académico, como en este caso, tener un trabajo.

Por otro lado, al establecer la relación entre las variables de estrés académico y rendimiento, se encontró que esta es indirecta pero no estadísticamente significativa. Por tanto, se puede afirmar que el estrés sí llega a afectar el rendimiento académico en los grupos, aunque, esta afectación no es tan grande como para que los estudiantes deban elegir una sola actividad, bien sea el trabajo o la universidad, pues se evidencia que los jóvenes tienen las habilidades y la disposición de tiempo necesaria para cumplir con sus responsabilidades académicas y laborales. 


\section{Referencias bibliográficas}

Almeida-Marquéz, L., y Lasluisa-Chacha, M. B. (2020). El estrés académico y su relación el rendimiento académico en una muestra de adolescentes (Tesis de pregrado). Pontificia Universidad Católica de Ecuador, Ecuador.

Ángeles I., y Gutiérrez, R. (2012). Acerca del afrontamiento y manejo del estrés. En Ángeles, I. y Gutiérrez, R. (Ed.), Estrés Organizacional (pp. $101-185)$. México: Editoriales Trillas.

Barraza-Macías, A. (2018). Inventario SIStémico COgnoscitivista para el estudio del estrés académico: Segunda versión de 21 ítems. Durango, México: Books-CECORFAN. Recuperado de http://www.ecorfan.org/libros/Inventario_SISCO_SV-

21/Inventario_sistémico_cognoscitivista_para_el_estudio_del_estrés.pdf

Barreto-Osma, D. A., Celis-Estupiñán, C. G. y Pinzón-Arteaga, I. A. (2019). Estudiantes universitarios que trabajan: subjetividad, construcción de sentido e in-satisfacción. Revista Virtual Universidad Católica del Norte, (58), 96-115.

Berrío-García, N., y Mazo-Zea, R. (2011). Estrés Académico. Revista de Psicología Universidad de Antioquia, 3(2), 65-82. Recuperado de http://pepsic.bvsalud.org/scielo.php?script=sci_arttext\&pid=S214548922011000200006\&lng=pt\&tlng=es

Caballero, C. (2006). Burnout, Engagement y Rendimiento Académico entre estudiantes Universitarios que trabajan y aquellos que no trabajan. Psicogente, 9(16). 11-27. Recuperado de http://revistas.unisimon.edu.co/index.php/psicogente/article/view/2678

Cáceres-Cáceres, G.S., \& Peña-Ballesteros, G.H. (2008). Validación del inventario SISCO del estrés académico y análisis comparativo en adultos jóvenes de la universidad industrial de Santander UIS y la Universidad Pontificia Bolivariana seccional Bucaramanga (Tesis de pregrado). Universidad Pontificia Bolivariana, Bucaramanga, Colombia.

Caldera-Montes, J.F., Pulido-Castro, B.E., y Martínez-González, M.G. (2007). Niveles de estrés y rendimiento académico en estudiantes de la carrera de Psicología del Centro Universitario de Los Altos. Revista de Educación y Desarrollo,4(7), 77-82. Recuperado de http://www.cucs.udg.mx/revistas/edu_desarrollo/anteriores/7/007_Caldera.pdf

Carrillo-Regalado, S., y Ríos-Almodóvar. (2013). Trabajo y rendimiento escolar de los estudiantes universitarios. el caso de la Universidad de Guadalajara, México. Revista de la educación superior, 13(2), 9-34.

Darolia, R. (2014). Working (and studying) day and night: Heterogeneous effects of working on the academic performance of full-time and part-time students. Economics of Educational Review, 38, 38-50. DOI: https://doi.org/10.1016/j.econedurev.2013.10.004

DeSimone, J. (2008). The Impact of Employment During School on College Student Academic Performance. National Bureau of Economic Research, Working Paper 14006, 1-38.

Domínguez-Castillo, R. A., Guerrero-Walker, G. J., y Domínguez-Castillo, J. G. (2015). Influencia del estrés en el rendimiento académico de un grupo de estudiantes universitarios. Educación y ciencia, 4(43), 31-40.

Edel-Navarro, R. (2003). El rendimiento académico: Concepto, Investigación y desarrollo. Revista Electrónica Iberoamericana sobre Calidad, Eficacia y Cambio en Educación, 1(2), 1-15. 
Francisco-Mendez, H. R. (2015). Estrés en adolescentes que estudian y trabajan (Tesis de Pregrado). Universidad Rafael Landívar. Ciudad de Guatemala, Guatemala. Recuperado de http://recursosbiblio.url.edu.gt/tesiseortiz/2015/05/22/Mendez-Hugo.pdf

Kloster-Kantlen, G. E., y Perrotta, F. D. (2019). estrés académico en estudiantes universitarios de la ciudad de Paraná. (Tesis de grado). Universidad Católica Argentina. Paraná, Argentina.

Neyt, B., Omey, E., Verhaest, D., Y Baert, S. (2019). Does Student Work Really Affect Educational Outcomes? A Review of the Literature. Journal of Economic Surveys, 33(3), 896-921. DOI: 10.1111/joes.12301.

Nonis, S. A., y Hudson, G. I. (2006). Academic Performance of College Students: Influence of Time Spent Studying and Working. Journal of Education for Bussines, 81(3), 151-159.

Maslach, C., Schaufeli, Y W., Leiter, M. (2001). Job Burnout. Annual Review of Psychology; ProQuest Medical Library, (52), 397-422.

Rivas-Acuña, V., Jiménez-Palma, C. C., Méndez-Méndez, H. A., Cruz-Arceo, M. A., Magaña-Castillo, M., y Victorino-Barra, A. (2014). Frecuencia e Intensidad del Estrés en Estudiantes de Licenciatura en Enfermería de la DACS. Horizonte Sanitario, 13(1), 162-169.

Saborío-Morales, L., e Hidalgo-Murillo, L. f. (2015). Síndrome de Burnout. Medicina Legal de Costa Rica, 32(1). ISSN 1409-0015.

Salinas-Quiroga, M. D., y González-Salazar, F. (2019). Influencia del trabajo sobre el promedio académico en estudiantes de odontología. magis, Revista Internacional de Investigación en Educación, 12(24), 41-52. doi: 10.11144/Javeriana.m12-24.itpa

Sotelo-García, E.E., y Gutiérrez-Luna, G.E. (2020). Relación entre estrés con el rendimiento académico en los alumnos del 2 nivel primaria del la institución educativa pública 22295. (Tesis de pregrado). Universidad autónoma de ICA, Perú.

Selye, H. (1956). The stress of life. New York, NY, US: McGraw-Hill.

\footnotetext{
Esta obra está bajo una Licencia Creative Commons

Attribución-NoCommercial 4.0 International
}

\section{(cc) EY-NC}

